\title{
DEVELOPMENT OF THE DIMPLE INSTABILITY ON LIQUID ${ }^{4} \mathrm{He}$
}

\author{
W. EBNER and P. LEIDERER \\ Fachbereich Physik der Technischen Universität München, 8046 Garching, West-Germany
}

\begin{abstract}
Electrons on the surface of liquid ${ }^{4} \mathrm{He}$ have been used to study the transition from a homogeneous two-dimensional charge distribution to the modulated charge density regime where the so-called dimple crystal is formed. The observation of hysteresis effects suggests that the transition is discontinuous.
\end{abstract}

Electrons localized on the surface of liquid helium have been investigated quite extensively as an example of a nearly ideal two-dimensional Coulomb system $[1,2]$. Recently it has been observed that the homogeneous spatial distribution of the surface charge can be destroyed by self-trapping of the electrons in macroscopic dimples, which appear when the electric field pulling the charges toward the surface exceeds a critical value [3]. These dimples, resulting from an electrohydrodynamic instability of the surface, were found to be arranged in a regular, hexagonal structure, with a lattice constant approximately given by the wavelength of the soft ripplon which drives the instability.

Theoretically the electrohydrodynamic instability of the electron-liquid helium system has first been considered by Gorkov and Chernikova. These authors suggested that above a critical electric field bandlike or hexagonal structures should appear on the helium surface, but the stability of such structures was an open question [2,4-6]. After the experimental verification of the dimple lattice Ikezi presented a theory which accounted for the observed stable hexagonal configuration and in addition predicted hysterestis effects at the transition from the homogeneous to the dimple state $[7]^{\neq 1}$. In the present work we report about an experimental investigation of this transition.

For the measurements we used an optical sample

\$1 A similar theory, however for a liquid surface without charges, has been developed by Kuznetsov and Spektor [8]. cell with glass plates at the top and bottom (diameter $8 \mathrm{~cm}$, distance $b=1.5 \mathrm{~cm}$ ) which also served as capacitor for applying the electric field. The lower plate, covered with a gold film, acted as a mirror, whereas the top plate had a transparent conductive coating of indium oxide and thus allowed to observe corrugations of the helium surface from above. The surface was located at a height $h \approx b / 2$ in most cases and was charged with electrons from a small filament.

Assuming that the surface is charged to saturation, the voltage $U$ applied to capacitor plates during the charging process determines the surface electron density $n=U / 4 \pi e h$. When the electric field after charging is increased at constant $n$, the surface is expected to become unstable at an applied voltage of

$U_{\mathrm{c}}=b\left[4 \pi(g \Delta \rho \sigma)^{1 / 2}-4 \pi^{2}(n e)^{2}\right]^{1 / 2}$,

where $\Delta \rho$ is the density difference between helium vapor and liquid, $\sigma$ is the surface tension and $g$ the acceleration of gravity. [In eq. (1) we have neglected a small correction term due to the polarizability of helium, and have assumed that the liquid level is at $h=$ $b / 2$.] The lowest critical voltage, $U_{\mathrm{cl}}=b\left(4 \pi^{2} g \Delta \rho \sigma\right)^{1 / 4}$, is obtained for the maximum charge density which in equilibrium can be held at the liquid surface, given by

$n_{\max } e=\left(g \Delta \rho \sigma / 4 \pi^{2}\right)^{1 / 4}$.

For the development of the instability two quite different regimes are found, depending on whether $m$ is above or below a threshold density $n_{\mathrm{t}}$ :

(a) $n_{\mathrm{t}}<n<n_{\max }$. As the voltage is slightly raised 
above $U_{c}$ and then held fixed some random surface indentation grows in amplitude, until charges leak through the surface at that point (probably forming the "bubblons" observed by Khaikin et al. [6]). $n$ then drops until the condition for instability (1) is no longer fulfilled, and the charge distribution becomes homogeneous and stable again. This process is observed repeatedly as the applied voltage is slowly increased further.

(b) $n<n_{\mathrm{t}}$. The situation completely changes as soon as the electron density has dropped below the threshold value $n_{\mathrm{t}}$ (or also if the initial surface electron density has been less than $n_{\mathrm{t}}$ ). Now the dimples which are forming at $U_{\mathrm{c}}$ grow only to a finite depth, and then arrange themselves in a stationary configuration.

The development of such a dimple "crystal" on liquid helium, as seen from the top, is illustrated in fig. 1 . The interval between the stages shown is about $2 \mathrm{~s}$. First, a wavelike structure appears at a somewhat arbitrary orientation (fig. 1ạ, upper left corner). Inhomogeneities of the electric field due to the side walls or slight misalignment of the capacitor plates may influence this first formation as well as residual vibrations which in spite of the vibrationally isolated cryostat may have reached the sample cell. In fig. $1 \mathrm{~b}$ the line structure has spread out further and the throughs begin to split up into dimples, showing the first indication of hexagonal symmetry. Finally, in fig. $1 \mathrm{c}$, the dimples completely fill the charged area. In this last stage a local redistribution of the dimples leads to a nearly perfect hexagonal lattice, with a small number of imperfections being "frozen in". The structure formed in this way is stable; small perturbations, like a slight vibration of the surface, do not destroy it.

The image plane in fig. 1 was chosen such that convex deformations of the surface, corresponding to local maxima, appear bright. The charge in each dimple is concentrated in the minimum, i.e. near the center of the individual hexagons. Although this lattice is reminiscent of the well-known Bénard structure which ap-

Fig. 1. Development of the dimple lattice on a ${ }^{4} \mathrm{He}$ surface charged with electrons from above. The pictures show the surface deformation approximately 2, 4 and 6 seconds after the voltage had been increased to $U_{\mathrm{c}}, 3345 \mathrm{~V}$ in this case. The temperature is $3.5 \mathrm{~K}$. The lattice constant agrees well with the wavelength of the soft ripplon (see ref. [3]).
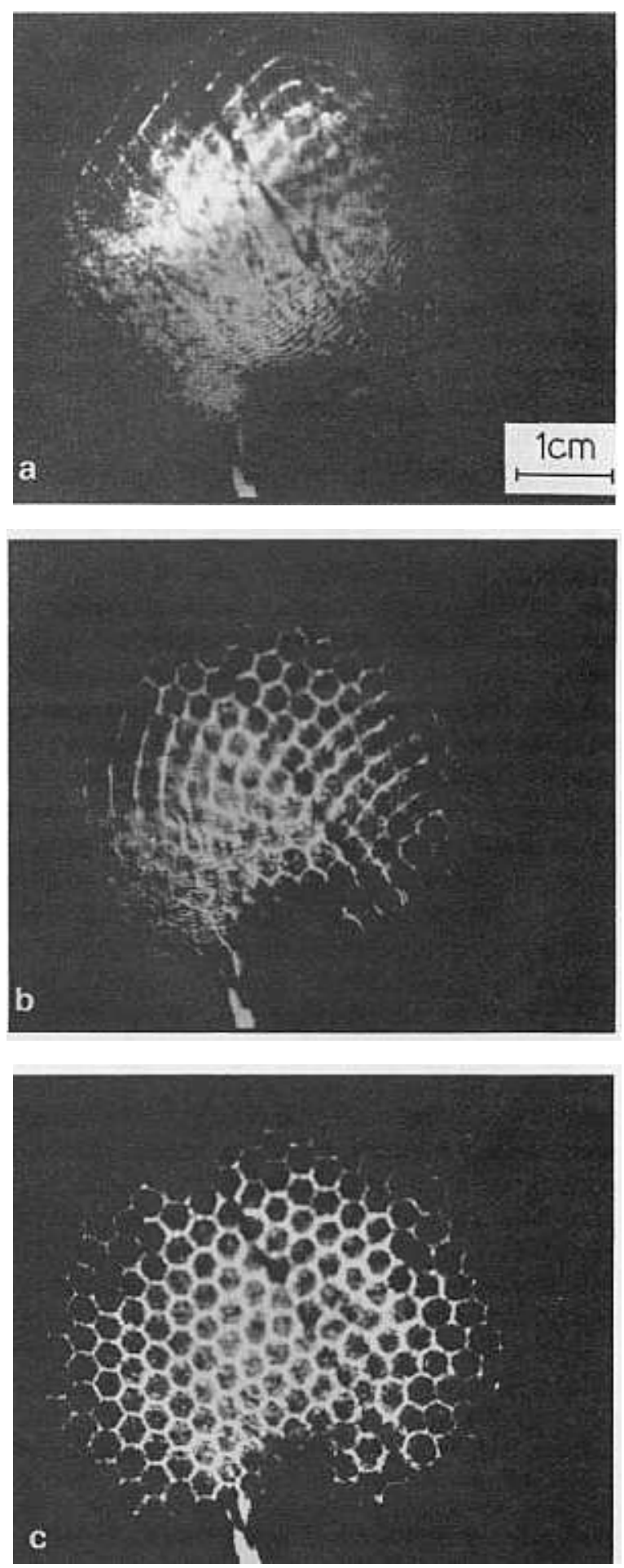
pears at the surface of liquids as a result of a convection instability [9], the physical situation is different because here the liquid is at rest once the dimples have formed.

The qualitative difference of the two instability regimes results from the magnitude of the electrostatic force acting upon the surface, which for $n<n_{t}-$ the stable deformation - is compensated by the restoring forces due to gravity and surface tension at a finite dimple depth, whereas for $n>n_{t}$ the electrostatic force always dominates. In the present experiment stable dimple lattices were observed to exist up to an estimated charge density of

$n / n_{\max }=0.08 \pm 0.03$,

a value comparable to, although somewhat lower than the limit $n_{\mathrm{t}} / n_{\max }=0.20$ given by Ikezi [7], and also smaller than the upper bound obtained in an earlier experiment [3]. When the surface charge density is close to $n_{t}$ the transition to the dimple lattice occurs at a very well defined applied voltage $U_{\mathrm{c} 2}$, as is demonstrated in fig. 2, where $U_{\mathrm{c} 2}$ is plotted versus temperature. According to eqs. (1)-(3) $U_{\mathrm{c} 2}$ is expected to vary as $(\Delta \rho \sigma)^{1 / 4}$, in excellent agreement with existing data on density and surface tension $[10,11]$. It is obvious from fig. 2 that cycling the system through the transi-

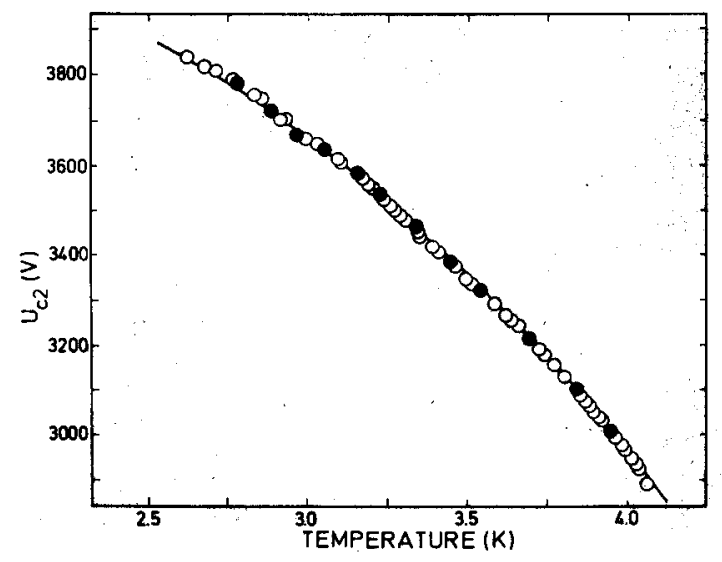

Fig. 2. Critical voltage $U_{\mathrm{c2}}$ for the formation of the dimple lattice on ${ }^{4} \mathrm{He}$ (distance of the capacitor plates $b=1.5 \pm 0.1$ $\mathrm{cm}$ ). In one run the surface was charged for each datum point to $n_{\max }$, and then $U$ was slowly increased until the dimples appearad at $U_{\mathrm{c} 2}$ (full symbols). Alternatively, the system was cycled through the transition several times before a datum point was taken (open symbols). The hysteresis $\Delta U$ is smaller than the symbol size. The full curve represents the variation of $(\Delta \rho \sigma)^{1 / 4}$ with temperature (refs. [10] and [11]). tion repeatedly without supplying additional charge does not change the critical voltage. Besides, visual inspection shows that the number of dimples also remains constant. The transition therefore apparently is reversible when $n<n_{\mathrm{t}}$.

Regarding the good reproducibility one is tempted to check for hysteresis effects in order to obtain information about the "order" of the transition. Hysteresis, although small in magnitude, was observed indeed. Once the dimple structure had developed at $U_{\mathrm{c} 2}$, the voltage could be reduced by $\Delta U \approx 5 \times 10^{-3} U_{\mathrm{c} 2}$, before the dimples disappeared and the homogeneous charge distribution was established again. We also determined the depth of the dimples from the interference pattern which results from a superposition of light reflected from the bottom and the top plate of the sample cell. At the transition the depth changes from $d=0$ to $d \approx 0.08 \mathrm{~mm}$ for the fully developed lattice as shown in fig. $1 \mathrm{c}$. Both $\Delta U$ and the discontinuity in $d$ at $U_{\mathrm{c} 2}$ are in an order-of-magnitude agreement with predictions by Ikezi [7] for an electron density $n=0.08 n_{\max }$. The drastically increasing hysteresis effects which according to the theory should appear for $n \rightarrow 0.20 n_{\max }$ could not be realized experimentally, either because close to this limit external perturbations easily lead to a premature loss of stability, or because higher order terms not considered in the theory reduce the range of $n$ where the surface is stable. Although for a better understanding certainly more detailed measurements are necessary, the observed hysteresis suggests that the formation of a dimple crystal can be regarded in analogy with a discontinuous, or first order, phase transition.

We gratefully acknowledge stimulating discussions with Dr. H. Ikezi and Professor V.B. Shikin. This work was supported by the Deutsche Forschungsgemeinschaft.

\section{References}

[1] M.W. Cole, Rev. Mod. Phys. 46 (1974) 451; C.C. Grimes, Surf. Sci. 73 (1978) 379.-

[2] V.B. Shikin and Yu.P. Monarkha, Fiz. Nizk. Temp. 1 (1975) 957 [Sov. J. Low Temp. Phys. 1 (1975) 459].

[3] M. Wanner and P. Leiderer, Phys. Rev. Lett. 42 (1979) 315;

P. Leiderer and M. Wanner, Phys. Lett. 73A (1979) 189.

[4] L.P. Gor'kov and D.M. Chernikova, Pis'ma Zh. Eksp. Teor. Fiz. 18 (1973) 119 [JETP Lett. 18 (1974) 68]. 
[5] L.P. Gor'kov and D.M. Chernikova, Dokl. Akad. Nauk SSR 228 (1976) 829 [Sov. Phys. Dokl. 21 (1976) 328].

[6] M.S. Khaikin, J. de Phys. C6 (1978) 1295.

[7] H. Ikezi, Phys. Rev. Lett. 42 (1979) 1688.

[8] E.A. Kuznetsov and M.D. Spektor, Zh. Eksp. Teor. Fiz. 71 (1976) 262 [Soy. Phys. JETP 44 (1976) 136].
[9] C. Normand, Y. Pomeau and M.G., Velarde, Rev. Mod. Phys. 49 (1977) 581.

[10] J. Wilks, The properties of liquid and solid helium (Claredon, Oxford, 1967).

[11] N. Devaraj and A.C. Hollis-Hallett, Can. J. Phys. 45 (1967) 2113. 